\title{
DESEMPENHO DE TILÁPIA DO NILO (OREOCHROMIS NILOTICUS) SUPLEMENTADA COM VITAMINA E
}

\author{
PERFORMANCE OF TILAPIA (OREOCHROMIS NILOTICUS) \\ SUPPLEMENTED WITHVITAMINE
}

\begin{abstract}
Navarro, R.D. ${ }^{1 *}$, Ferreira, W.M. ${ }^{2}$, Ribeiro Filho, O.P. ${ }^{3}$, Veloso, D.P. ${ }^{4}$, Fontes, D.O. ${ }^{2}$ e Silva, R.F.
1Unifenas. Faculdade de Zootecnia. Rodovia MG/179, km 0. Campus Universitário. CEP 37130000. Alfenas, MG. Brasil. rddnavarro@yahoo.com.br

${ }^{2}$ Departamento de Zootecnia. Escola de Veterinária. Av. Antônio Carlos, 6627. C.P. 567. Campus da Universidade Federal de Minas Gerais. CEP 30 123-970. Belo Horizonte, MG. Brasil. *rddnavarro@yahoo.com.br ${ }^{3}$ Departamento de Biologia. Universidade Federal de Viçosa. Brasil.

${ }^{4}$ Departamento de Química. Universidade Federal de Minas Gerais. Brasil.
\end{abstract}

\section{PalaVRas chaVe adicionais}

Exigência nutricional. Ácidos graxos. Nutrição de peixes.

\section{RESUMO}

Objetivou-se avaliar a suplementação de Vitamina $\mathrm{E}$ no desempenho e qualidade de carcaça de tilápia do Nilo (Oreochromis niloticus). Os experimentos foram conduzidos no ranário experimental do Departamento de Biologia Animal, UFV, Viçosa, Minas Gerais, Brasil, no período de 09/01/ 2005 a 25/04/2005, totalizando 106 dias. Foram utilizadas 400 pós larvas revertidas (Oreochromis niloticus) em um experimento com um delineamento inteiramente ao acaso com cinco tratamentos $(0$, $50,100,150$ e $200 \mathrm{mg} / \mathrm{kg}$ de vitamina E monofosfato) numa ração isoproteíca $36 \%$ de $\mathrm{PB}$ e isocalórica $3600 \mathrm{kcal}$ de ED/kg com quatro repetições. Não se observou efeito significativo para peso final, para ganho de peso total, para ganho de peso diário dos animais, para taxa de eficiência protéica e para taxa de sobrevivência. Para o índice viscerossomático houve diferença significativa para o tratamento sem suplementação, com $100 \mathrm{mg}$ e com $150 \mathrm{mg} / \mathrm{kg}$ desta vitamina. Para o ácido graxo oléico (C18:1) a utilização do tratamento sem vitamina $E$ foi significamente maior em relação aos outros tratamentos. O ácido graxo eicosapentaenóico EPA (C20:5 13 ) foi estatisticamente significativo para o tratamento sem suplementação

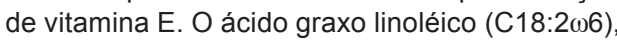
ácido graxo $\gamma$-linolênico (C18:3 $\omega 6)$, ácido graxo $\alpha$ -

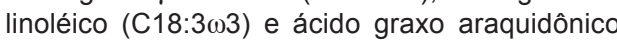

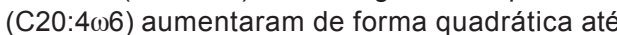

Recibido: 6-2-08. Aceptado: 17-9-08.

\section{AdDitionAL KEYWORDS}

Nutritional requirement. Fatty acids. Nutrition of fish.

o nível estimado de $110 \mathrm{mg}, 111 \mathrm{mg}, 113$ mg e 140 $\mathrm{mg}$ de vitamina $\mathrm{E}$ por $\mathrm{kg}$ de ração, respectivamente.

\section{SUMMARY}

The effect of vitamin $\mathrm{E}$ supplementation on performance and carcass quality of Nile tilapia (Oreochromis niloticus). The research was carried out in the experimental frog raising facilities at the Department of Animal Biology, UFV, Minas Gerais state, Brazil. Four hundred post larva reversed (Oreochromis niloticus) were used for studies. The experiments were randomly designed with five treatments (supplying 0,50 , 100,150 , and $200 \mathrm{mg} / \mathrm{kg}$ of vitamin $\mathrm{E}$ monophosphate) in an isoproteic ration with $36 \% \mathrm{CP}$ and isocaloric with $3600 \mathrm{kcal} / \mathrm{kg}$ of DE with four repetitions. It was not observed significant effect for final weight, total weight gain, daily weight gain, protein efficiency rate and survivor rate. The viscerosomatic index showed significant differences for the treatment without vitamin $\mathrm{E}$ supplementation and with 100 and $150 \mathrm{mg}$ of this vitamin. For oleic fatty acid, the treatment without vitamin E was significantly higher. The eicosapentaenoic fatty acid (C20:5 03 ) was statistically significant for the treatment without vitamin $\mathrm{E}$. The

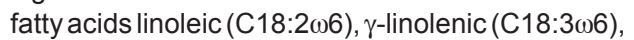

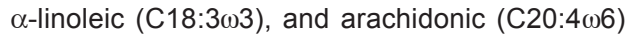


increased in a quadratic way up to the estimated levels of $110 \mathrm{mg}, 111 \mathrm{mg}, 113 \mathrm{mg}$, and $140 \mathrm{mg}$ of vitamin $\mathrm{E} / \mathrm{kg}$ of ration, respectively.

\section{INTRODUÇÃO}

A tilápia Oreochromis niloticus, é considerada o peixe mais importante do século XXI, cultivado em mais de 100 países e também no Brasil devido sua rusticidade e rápido crescimento, sendo a sua carne considerada de ótima qualidade (Lovshin, 1997, Tachibana et al., 2000). O interesse pelo cultivo dessa espécie, no Sul e Sudoeste do país, cresceu rapidamente nos últimos oito anos em virtude da tecnologia de reversão sexual e da pesca esportiva representada pelos pesque-pagues. A tilápia é criada em diversos sistemas, desde a cultura semiintensiva em tanques que recebem dejetos animais, como em cultivos intensivos em raceways e tanques-rede (Bhujel, 2000).

Nos sistemas de cultivo intensivo, em que a única fonte de nutrientes é a ração, a deficiência vitamínica pode ser observada, quadro que ganha importância durante a criação em tanque-rede ou culturas em raceway (Pezzato, 1999).

O desenvolvimento eficiente e saudável dos animais passa obrigatoriamente pelo fornecimento de uma dieta que satisfaz as necessidades básicas de crescimento, contendo concentrações próximas do ideal e seus diversos componentes, aliados a tecnologia de preparação. A estocagem, a concentração de vitaminas e minerais, a biodisponibilidade dos nutrientes são exemplos de parâmetros que interferem no desenvolvimento do animal (Cavalheiro e Pereira, 1998; Navarro et al., 2007).

Entre os micronutrientes, a vitamina E, considerada nome genérico, descreve as bioatividades de derivados de tocoferois e tocotrienois, que são vitaminas lipossolúveis e possuem alto poder antioxidante. Os quatro isômeros dos tocotrienois $(\alpha-\mathrm{T} 3, \beta-\mathrm{T} 3, \gamma$ $\mathrm{T} 3$ e $\delta$ - T3) são estruturalmente relacionados aos seus correspondentes homólogos dos tocoferóis $(\alpha-\mathrm{T}, \beta-\mathrm{T}, \gamma$-T e $\delta$-T), mas diferem nas suas cadeias laterais nas quais os isômeros contêm três duplas ligações (Almeida et al., 2006).

Segundo Barreto (1998), os compostos com atividade de vitamina E são de baixa atividade quando ocorrem naturalmente nos alimentos. Porém, entre esses, o d- $\alpha$ tocoferol é o composto natural com maior atividade biológica da vitamina E presente nos alimentos.

O $\alpha$-tocoferol é o representante mais importante do grupo de substância com atividade de vitamina E. Apresenta maior atividade biológica quando comparado aos demais compostos, devido ao maior índice de absorção intestinal, maior deposição nos tecidos, menor excreção fecal, além de ser oxidado mais lentamente (Barreto, 1998, Sampaio et al., 2004).

De todas as vitaminas dadas aos peixes, a vitamina $E$ tem sido amplamente estudada em dietas de peixes. Isso, porque, é a vitamina que possui papel importante no metabolismo animal por possuir propriedades antioxidantes (Thakur e Srivastava, 1996 e Guerra et al., 2004). O requerimento de vitamina E tem sido relatado por alguns autores como Murray e Andrews, (1994) e Watanabe e Takashima (1977) para carpa, Wilson et al. (1984) para channel catfish, Kocabas e Gatlin (1999) para Morone chrysops female $\times M$. saxatilis male, Paul et al. (2004) para Cirrhinus mrigala, Shiau e Shiau (2001), Huang et al. (2004) e Fogaça e Sant'ana (2007) para tilápia do Nilo. Além disso, poucas informações têm referenciado as exigências nutricionais e qualidade de carcaça. Nesse sentindo, objetivou-se avaliar a suplementação de Vitamina E no desempenho e na qualidade de carcaça de tilápia do Nilo (Oreochromis niloticus).

\section{MATERIALEMÉTODOS}

Os experimentos foram conduzidos no ranário experimental do Departamento de Biologia Animal, Universidade Federal de 


\section{DESEMPENHO DE TILÁPIA DO NILO COM VITAMINAE}

Viçosa, Minas Gerais, Brasil, no período de 09/01/2005 a 25/04/2005, no total de 106 dias.

Foram utilizadas 400 pós-larvas revertidas (Oreochromis niloticus), provenientes de uma empresa que produz alevinos, com peso e comprimento inicial de $1,40 \pm 0,88 \mathrm{~g}$

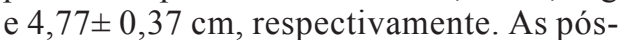
larvas foram distribuídas em 20 aquários com capacidade de 1000 litros e com renovação constante de água $7,5 \mathrm{ml} / \mathrm{minu}-$ to, seguindo um delineamento inteiramente ao acaso com cinco tratamentos $(0,50,100$, 150 e $200 \mathrm{mg} / \mathrm{kg}$ de vitamina E monofosfato - D alfa tocoferol) numa ração isoproteíca $36 \%$ de $\mathrm{PB}$ e isocalórica $3600 \mathrm{kcal}$ de $\mathrm{ED} / \mathrm{kg}$ (tabela I), com quatro repetições. A fase de adaptação foi de cinco dias.

Tabela I. Composições percentuais, químicas e calculadas das dietas experimentais. (Percentagem and chemical composition of experimental diets as fed).

\begin{tabular}{|c|c|c|c|c|c|}
\hline \multirow[b]{2}{*}{ Ingrediente (\%) } & \multicolumn{5}{|c|}{ Vitamina E mg/kg } \\
\hline & 0 & 50 & 100 & 150 & 200 \\
\hline Farelo de soja & 21,50 & 21,50 & 21,50 & 21,50 & 21,50 \\
\hline Glúten de milho & 30,00 & 30,00 & 30,00 & 30,00 & 30,00 \\
\hline Milho & 28,50 & 28,50 & 28,50 & 28,50 & 28,50 \\
\hline Farinha de peixe & 9,00 & 9,00 & 9,00 & 9,00 & 9,00 \\
\hline Óleo de soja & 7,60 & 7,60 & 7,60 & 7,60 & 7,60 \\
\hline Fosfato bicálcio & 1,37 & 1,37 & 1,37 & 1,37 & 1,37 \\
\hline Calcário & 0,51 & 0,51 & 0,51 & 0,51 & 0,51 \\
\hline Sal & 0,40 & 0,40 & 0,40 & 0,40 & 0,40 \\
\hline Premix vitamínico e mineral & 0,60 & 0,60 & 0,60 & 0,60 & 0,60 \\
\hline Metionina & 0,34 & 0,34 & 0,34 & 0,34 & 0,34 \\
\hline Lisina & 0,15 & 0,15 & 0,15 & 0,15 & 0,15 \\
\hline BHT (antioxidante) & 0,02 & 0,02 & 0,02 & 0,02 & 0,02 \\
\hline Vitamina E mg/kg & 0 & 50 & 100 & 150 & 200 \\
\hline \multicolumn{6}{|l|}{ Composição calculada\% } \\
\hline Proteína bruta ${ }^{1}$ & 36 & 36 & 36 & 36 & 36 \\
\hline Energia digestível ${ }^{2} \mathrm{kcal} / \mathrm{kg}$ & 3600 & 3600 & 3600 & 3600 & 3600 \\
\hline Fibra bruta ${ }^{1}$ & 2,30 & 2,30 & 2,30 & 2,30 & 2,30 \\
\hline Lisina & 1,50 & 1,50 & 1,50 & 1,50 & 1,500 \\
\hline Metionina + cistina & 1,11 & 1,11 & 1,11 & 1,11 & 1,11 \\
\hline Treonina & 1,35 & 1,35 & 1,35 & 1,35 & 1,35 \\
\hline Triptófano & 0,29 & 0,29 & 0,29 & 0,29 & 0,29 \\
\hline Cálcio & 1,04 & 1,04 & 1,04 & 1,04 & 1,04 \\
\hline Fósforo total ${ }^{2}$ & 0,80 & 0,80 & 0,80 & 0,80 & 0,80 \\
\hline Relação ED:PB & 10 & 10 & 10 & 10 & 10 \\
\hline
\end{tabular}

${ }^{1}$ Com base nas análises de laboratório LNA/DZO.

Baseados nos valores propostos pelo NRC (1993) e por Rostagno et al. (2000).

${ }^{3}$ Premix vitamínico comercial (5 kg/t), com níveis de garantia por quilograma de produto: Vit. A, 1200000 UI; Vit. $D_{3}, 200000$ UI; Vit k, 2400 mg; Vit B ${ }_{3}, 4800$ mg; Vit $B_{2}, 4800$ mg, Vit $B_{6}, 4000$ mg, Vit $B_{12}, 4800$ mg, ác. Fólico, $1200 \mathrm{mg}$; pantotenato Ca $12000 \mathrm{mg}$; Vit. C, $48000 \mathrm{mg}$; biotina, $48 \mathrm{mg}$; cloreto de colina, 108000 mg; niacina, $24000 \mathrm{mg}$; e premix mineral comercial $(1 \mathrm{~kg} / \mathrm{t})$, com níveis de garantia por quilograma do produto: Fe, 50000 mg; Cu, 3000 mg; 20000 mg; Mn, 20000 mg; Zn, 3000 mg; I, 100 mg; Co, 10 mg; Se, $100 \mathrm{mg}$. 
As dietas experimentais foram peletizadas, secas em estufa de circulação forçada a $55^{\circ} \mathrm{C}$ e, posteriormente, fracionadas nos diâmetros de $1 \mathrm{~mm}$, entre $4 \mathrm{~mm}$ a $6 \mathrm{~mm}$, sendo utilizadas de acordo com o tamanho dos peixes. As dietas permaneceram armazenadas a $-20^{\circ} \mathrm{C}$ até sua utilização. Os animais foram alimentados de forma controlada em três refeições ( $08 \mathrm{~h} 00 \mathrm{~min}, 13 \mathrm{~h} 00 \mathrm{~min}$ e $17 \mathrm{~h} 00 \mathrm{~min}$ ) e a oferta de ração foi de $5 \%$ do peso vivo, sendo ajustada a cada 15 dias. Foram realizadas despescas através de rede de malha de três mm, sendo capturado $15 \%$ dos animais. As biometrias foram realizadas com auxílio de paquímetro e de balança de precisão. Os aquários foram sifonados, diariamente, para retirar sobras de ração e fezes. $\mathrm{O}$ fotoperíodo foi de 12 horas. A medição da temperatura da água foi realizada diariamente, às $7 \mathrm{~h} 00 \mathrm{~min}$ e às $17 \mathrm{~h} 00 \mathrm{~min}$, enquanto o pH e o oxigênio dissolvido foram medidos a cada sete dias.

No final do experimento, após jejum de 24 horas, os peixes foram abatidos por insensibilização (caixas isotérmicas com gelo moído e água na proporção $1: 1$ ) de acordo com (Navarro et al., 2009). Depois do abate, foram eviscerados. Para realizar as análises bromatológicas, as carcaças foram secas em estufa com ventilação forçada a $55^{\circ} \mathrm{C}$ por $48 \mathrm{~h}$. Depois da secagem, passaram por uma moagem em moinho de bola, até formar um pó com granulometria homogênea.

No inicio do experimento, cinqüenta peixes foram abatidos para posterior análise de carcaça. Depois do abate, as carcaças foram pesadas em balança de precisão 0,001 g com o objetivo de analisar e de determinar a composição inicial da carcaça em teor de água, proteína e extrato etéreo. O extrato etéreo foi feito com hidrólise ácida prévia. As análises dos ingredientes utilizados nas dietas e das amostras dos peixes foram realizadas no laboratório de Nutrição Animal do Departamento de Zootecnia (LNA/DZO) da Universidade Federal de Minas Gerais, Minas Gerais, Brasil, conforme procedimentos descritos por AOAC (1995).
Para determinação do perfil de ácidos graxos, utilizou-se o gás Cromatografo Varian CP-3380 e uma Coluna: DB-WAX 25 $\mathrm{m} \times 0,25 \mathrm{~mm} \times 0,25 \mathrm{~mm}$ (Jew Scientific) do Departamento de Química da Universidade Federal de Minas Gerais - UFMG. A gordura extraída foi esterificada (produção de ésteres metílicos) segundo metodologia desenvolvida pelo STQ (1998). Obtidos os ésteres metílicos, foram analisados em cromatógrafo gasoso. O hidrogênio foi utilizado como gás de arraste, em $40 \mathrm{~cm} / \mathrm{seg}$. A temperatura inicial da coluna, de $50^{\circ} \mathrm{C}$ por 2 minutos, elevou-se $4^{\circ} \mathrm{C}$ por minuto, até $220^{\circ} \mathrm{C}$, permanecendo nessa temperatura por mais de 25 minutos. A temperatura do injetor foi de $260^{\circ} \mathrm{C}$, e a do detector $260^{\circ} \mathrm{C}$. Foram injetados $2 \mu \mathrm{l}$ de amostra e utilizaram-se padrão de ácidos graxos Sigma 189-19.

Foram analisados os parâmetros nutricionais de ganho de peso (GP), taxa de crescimento específico (TE), taxa de sobrevivência (TS), a composição química da carcaça (extrato etéreo e proteína), a porcentagem de proteína no ganho de peso (\%PBGP), a porcentagem de gordura no ganho de peso (\%GGP), o índice hepatossomático (IHS), o índice viscerossomático (IVS).

Ganho de peso dos peixes (GP) foi calculado pela diferença dos pesos médios da parcela final e inicial

$\mathrm{GP}=$ peso final $(\mathrm{g})$ - peso inicial $(\mathrm{g})$

Taxa de eficiência protéica

$\mathrm{TEP}=\mathrm{GP}(\mathrm{g}) /$ proteína ingerida $(\mathrm{g})$

Porcentagem de proteína no ganho de peso

$\% \mathrm{PBGP}=$ Proteína corporal final $(\mathrm{g})$ proteína corporal inicial (g)/ganho de peso (g)

Porcentagem de gordura no ganho de peso

$\% \mathrm{GGP}=$ Gordura corporal final $(\mathrm{g})$ - gordura corporal inicial (g)/ganho de peso (g) Índice viscerossomático (IVS)

$\mathrm{IVS}=\mathrm{PV} / \mathrm{PC} \times 100$

$\mathrm{PC}=$ peso corporal

$\mathrm{PV}=$ peso da víscera 


\section{DESEMPENHO DE TILÁPIA DO NILO COM VITAMINAE}

Índice hepatossomático (IHS)

$\mathrm{IHS}=\mathrm{PF} / \mathrm{PC} \times 100$

$\mathrm{PC}=$ peso corporal

$\mathrm{PF}=$ peso do fígado

A taxa de crescimento específico foi determinada pela fórmula $\mathrm{TCE}=(\ln )$ peso total final - (ln) peso total inicial dividido pelo tempo de experimento.

As análises estatísticas foram realizadas pelo programa SAS (1997). Os efeitos da suplementação de vitamina $\mathrm{E}$ foi analisado aplicando o teste de Duncan com 5\% de probabilidade como teste de médias. Também foram utilizados modelos de regressão linear, quadrática, com base na significância dos coeficientes de regressão aplicando teste no coeficiente de determinação, na soma de quadrado dos desvios e nos fenômenos em estudo.

\section{RESULTADOSEDISCUSSÃO}

Valores médios obtidos de temperatura foi de 28,23 $\pm 0,63 ; 7,25 \pm 0,58$ para $\mathrm{pH} ; 5,23$ $\pm 0,85 \mathrm{mg} \mathrm{l}^{-1}$ para oxigênio dissolvido. Este ambiente permaneceu dentro das condições ótimas para crescimento da espécie de acordo com (Bhujel, 2000).
Os resultados de peso final, de ganho de peso, de ganho de peso diário, de taxa de eficiência protéica, de taxa de crescimento específico e de taxa de sobrevivência são apresentados na tabela II.

Não foi observado efeito significativo $(p>0,05)$ da suplementação de vitamina $E$ para peso final (PF), para ganho de peso total (GP) e para ganho de peso médio diário dos animais (GPD). Segundo Martins et al., (2008) a vitamina E (alfa-tocoferol) é a principal vitamina lipossolúvel responsável por proteger a membrana celular da peroxidação lipídica (Thakur e Srivastava, 1996). Sua deficiência causa baixo crescimento, exoftalmia, ascite, anemia, brânquias pálidas e disformes, prejuízo à produção de eritrócitos, menor resposta de anticorpo, despigmentação e acúmulo de gordura no fígado (Martins et al., 2008).

Os resultados de desempenho em tilápia estão de acordo com Wilson et al. (1984) que não observaram diferença significativa para ganho de peso, ao suplementarem vitamina E para catfish. Resultados superiores foram observados por Kocabas e Gatlin (1999), que encontraram maior ganho de peso utilizando $20 \mathrm{mg}$ por $\mathrm{kg}$ de vitamina $\mathrm{E}$

Tabela II . Peso final ( $g$ ) (PF), ganho de peso (GP) ganho de peso diário (GPD), taxa de eficiência protéica (TEP), taxa de crescimento especifico (TCE), taxa de sobrevivência (TS) de alevinos de tilápia alimentados com dietas com suplementação de vitamina E na ração. (Final weight (g) (PF), weight gain, (PG) daily weight gain (GPD), protein efficiency rate (TEP), specific growth rate (TCE), survival rates (TS) of fingerlings of tilapia fed diets with supplementation of vitamin $E$ in the diet).

\begin{tabular}{lcccccr}
\hline & \multicolumn{7}{c}{ Vitamina E mg/kg } \\
& 0 & 50 & 100 & 150 & 200 & C.V. \\
\hline PF $(\mathrm{g})$ & $74,04 \pm 5,53$ & $71,42 \pm 5,93$ & $73,87 \pm 6,22$ & $72,72 \pm 3,18$ & $72,52 \pm 4,47$ & 15,67 \\
GP $(\mathrm{g})$ & $72,62 \pm 5,45$ & $69,89 \pm 5,94$ & $72,40 \pm 6,23$ & $71,31 \pm 3,20$ & $71,04 \pm 4,46$ & 17,36 \\
GPD & $0,62 \pm 0,05$ & $0,61 \pm 0,05$ & $0,63 \pm 0,05$ & $0,62 \pm 0,02$ & $0,62 \pm 0,03$ & 17,83 \\
TEP & $2,05 \pm 0,31$ & $1,94 \pm 0,36$ & $2,05 \pm 0,36$ & $1,98 \pm 0,31$ & $1,98 \pm 0,36$ & 17,31 \\
TCE & $0,030 \pm 0,0$ & $0,030 \pm 0,0$ & $0,032 \pm 0,0$ & $0,032 \pm 0,0$ & $0,030 \pm 0,0$ & 10,37 \\
TS & $96,6 \pm 5,7$ & $98,33 \pm 2,8$ & $98,33 \pm 2,88$ & $100,00 \pm 0,0$ & $100,00 \pm 0,0$ & 3,20 \\
\hline
\end{tabular}

Letras distintas na mesma linha indicam diferença significativa $(p<0,05)$ pelo teste de Duncan. Valores em medias \pm erro padrão C.V. (coeficiente de variação). 
para a espécie Morone chrysops female $\times M$. saxatilis male. Shiau e Shiau (2001) observaram melhor desempenho para juvenis de tilápia do Nilo com $40 \mathrm{mg}$ de vitamina E. Já Paul et al. (2004) observaram, em Cirrhinus mrigala que houve melhora no desempenho utilizando $120 \mathrm{mg} / \mathrm{kg}$ de vitamina E para peso final e para ganho de peso.

Não se verificou significância $(p>0,05)$ na taxa de eficiência protéica (TEP) em razão do aumento da suplementação de vitamina E. Outros autores, trabalhando com diversas espécies, verificaram a tendência de aumento na TEP com o aumento da suplementação de vitamina E (Kocabas e Gatlin, 1999; Paul et al., 2004).

Não foram observadas diferenças significativas $(p>0,05)$ para taxa de crescimento especifico e para taxa de sobrevivência (TS). Baker e Davies (1996) e Bai e Lee (1998), observaram que a vitamina $\mathrm{E}$ melhora o sistema imune dos animais atuando como antioxidantes. Os resultados descritos neste estudo para tilápia estão de acordo com os estudos de Kocabas e Gatlin (1999) e Paul et al. (2004), que não observaram diferença significativa para taxa de sobrevivência. Os mesmos autores relataram que houve maior taxa de crescimento específico, ao utilizar $120 \mathrm{mg} / \mathrm{kg}$ de vitamina E para Cirrhinus mrigala.

O efeito da suplementação de vitamina $\mathrm{E}$ sobre a matéria seca, porcentagem de proteína e gordura da carcaça, sobre a porcentagem de proteina no ganho de peso, porcentagem de gordura no ganho de peso e sobre o índice viscerossomatico, encontrase na tabela III.

Os níveis de suplementação de vitamina E na dieta não influenciaram ( $p>0,05)$ o teor de matéria seca, na \%PB, \%PBGP. Resultado semelhante para proteína bruta foi observado por Ruff et al. (2003), Paul et al. (2004), Wing-Keong et al. (2004) e Fogaça e Sant'Ana (2007). Para porcentagem de EE na carcaça e gordura no ganho de peso não foi observado diferença significativa. Esses resultados estão de acordo com os achados no estudo de Ruff et al. (2003), Paul et al. (2004) e Fogaça e Sant'Ana (2007).

Não foram observadas diferenças significativas $(\mathrm{p}>0,05)$ para o índice hepatossomático. Entretanto, o índice viscerosso-

Tabela III. Composição em matéria seca (MS), proteína bruta (PB), extrato etéreo (EE), proteina no ganho de peso (PBGP), gordura no ganho de peso $(G G P)$, índice viscerosomático (IVS), índice hepatossomático (IHS) de carcaça de alevinos de tilápia alimentados com dietas com suplementação de vitamina E na ração. (Dry matter (MS), crude protein (PB), fat (EE), weigth gain protein (PBGP), weigth gain fat (GGP), viscera-somatic index (IVS), liver somatic index (IHS) of fingerlings of tilapia fed diets with supplementation of vitamin $E$ in the diet).

\begin{tabular}{lcccccr}
\hline \multicolumn{7}{c}{ Vitamina E mg/kg } \\
& 0 & 50 & 100 & 150 & 200 & C.V. \\
\hline MS \% & $27,97 \pm 3,20$ & $28,77 \pm 0,59$ & $28,46 \pm 1,43$ & $28,47 \pm 2,36$ & $27,73 \pm 3,92$ & 2,45 \\
PB \% & $54,67 \pm 0,12$ & $54,51 \pm 0,08$ & $55,45 \pm 0,20$ & $54,77 \pm 0,89$ & $55,00 \pm 2,62$ & 2,29 \\
EE \% & $17,81 \pm 2,0$ & $19,74 \pm 1,59$ & $21,25 \pm 3,04$ & $17,16 \pm 1,69$ & $20,55 \pm 2,98$ & 11,27 \\
PBGP $\%$ & $53,82 \pm 0,15$ & $53,63 \pm 0,15$ & $54,57 \pm 0,28$ & $53,88 \pm 0,90$ & $54,10 \pm 2,69$ & 2,37 \\
GGP $\%$ & $17,69 \pm 3,19$ & $19,62 \pm 0,71$ & $19,90 \pm 1,41$ & $17,40 \pm 2,96$ & $20,39 \pm 3,93$ & 11,90 \\
IHS & $1,20 \pm 0,09$ & $1,24 \pm 0,45$ & $1,31 \pm 0,31$ & $1,27 \pm 0,23$ & $1,11 \pm 0,12$ & 17,16 \\
IVS & $9,40 \pm 1,66^{\text {a }}$ & $8,75 \pm 0,99^{\text {b }}$ & $9,28 \pm 2,02^{\text {a }}$ & $9,42 \pm 1,31^{\text {a }}$ & $8,28 \pm 1,76^{\text {b }}$ & 26,64 \\
\hline
\end{tabular}

Letras distintas na mesma linha indicam diferença significativa $(p<0,05)$ pelo teste de Duncan. Valores em medias \pm erro padrão C.V. (coeficiente de variação).

Archivos de zootecnia vol. 59, núm. 226, p. 190. 


\section{DESEMPENHO DE TILÁPIA DO NILO COM VITAMINAE}

mático foi significativamente maior $(\mathrm{p}<0,05)$ para o tratamento sem suplementação de vitamina E, com $50 \mathrm{mg}$, com $100 \mathrm{mg}$ e $150 \mathrm{mg}$ desta vitamina em relação ao tratamento com $200 \mathrm{mg} / \mathrm{kg}$, sendo essa tendência também observada para peso final referido neste estudo. Esses resultados podem ser explicados pelo estudo de Shearer (1994), onde observou que, o tamanho relativo dos tecidos e órgãos, sob condições adequadas de nutrição, é dependente do tamanho do peixe. Contrapondo-se ao relatado no estudo, Tocher et al. (2002), não observaram diferença significativa para IHS. No resultado obtido por Pearce et al. (2003), foi observado que em Oncorhynchus mykiss houve melhor desenvolvimento do IHS no tratamento sem suplementação de vitamina E.

$\mathrm{O}$ efeito da suplementação de vitamina $\mathrm{E}$ sobre ácidos graxos poliinsanturados encontra-se na tabela IV.

Para o ácido graxo oléico (C18:1), a utilização do tratamento sem vitamina $\mathrm{E}$ foi significamente $(\mathrm{p}<0,05)$ maior (tabela IV). A suplementação de vitamina $\mathrm{E}$ influenciou $(\mathrm{p}<0,05)$ a porcentagem de ácido graxo

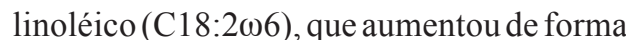
quadrática até o valor estimado de suplementação de $110 \mathrm{mg}$ de vitamina $\mathrm{E} / \mathrm{kg}$ de ração (tabela IV). Essa tendência também foi observado para o ácido graxo $\gamma$-linolênico

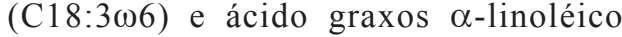

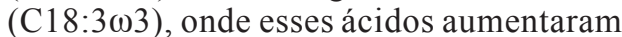
de forma quadrática até a suplementação de 111 e 113 mg de vitamina E por kg de ração, respectivamente (tabela IV). Resultados semelhantes foram observados por Bai e Lee (1998) que verificaram um aumento do

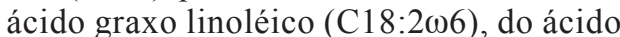

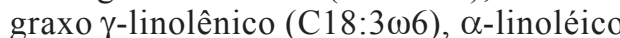
(C18:3w3), com aumento dos níveis de vitamina E. Com base nos dados obtidos neste estudo, pode-se inferir que essa maior quantidade de ácidos linoléico e linolênico é devida à capacidade da vitamina $\mathrm{E}$ de agir contra os radicais livres, neutralizando antes que possam oxidar lipídeos insaturados de membranas. Tocher et al. (2002) analisaram o efeito da Vitamina E nos ácidos graxos no fígado e concluíram que para a espécie Scophthalmus maximus os níveis de vitamina E não influenciaram os ácidos

Tabela IV. Ácidos graxos polissanturados da carcaça de tilápia alimentadas com suplementação de vitamina $E$. (Fatty acids polyunsaturated the carcass of tilapia fed supplementation of vitamin E).

\begin{tabular}{|c|c|c|c|c|c|c|}
\hline \multirow[b]{2}{*}{ Ácidos graxos } & \multirow[b]{2}{*}{0} & \multicolumn{3}{|c|}{ Vitamina E mg/kg } & \multirow[b]{2}{*}{200} & \multirow[b]{2}{*}{ C.V. } \\
\hline & & 50 & 100 & 150 & & \\
\hline C18:1 & $34,08 \pm 1,33^{a}$ & $32,52 \pm 0,92^{\mathrm{ab}}$ & $27,56 \pm 0,12^{c}$ & $25,31 \pm 0,77^{d}$ & $31,02 \pm 0,93^{b}$ & 3,01 \\
\hline C18:2 $2 \omega 6^{*}$ & $13,29 \pm 0,62^{\mathrm{d}}$ & $14,97 \pm 1,55^{c}$ & $27,72 \pm 0,05^{\mathrm{a}}$ & $25,66 \pm 0,05^{b}$ & $14,00 \pm 0,32^{\mathrm{dc}}$ & 3,98 \\
\hline 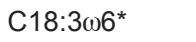 & $0,27 \pm 0,02^{\mathrm{d}}$ & $0,37 \pm 0,04^{c}$ & $1,10 \pm 0,0^{\mathrm{a}}$ & $0,90 \pm 0,0^{\mathrm{b}}$ & $0,36 \pm 0,01^{c}$ & 3,93 \\
\hline C18:3 $\omega 3^{*}$ & $0,60 \pm 0,11^{d}$ & $0,79 \pm 0,08^{c}$ & $2,04 \pm 0,0^{a}$ & $1,80 \pm 0,01^{b}$ & $0,72 \pm 0,01^{c}$ & 5,50 \\
\hline$C 20: 4 \omega 6^{*}$ & $0,00^{\mathrm{c}} \mathrm{ND}$ & $0,00^{\mathrm{c}} \mathrm{ND}$ & $0,29 \pm 0,02^{\mathrm{a}}$ & $0,29 \pm 0,03^{a}$ & $0,16 \pm 0,04^{b}$ & 17,3 \\
\hline$C 20: 5 \omega 3$ & $0,50 \pm 0,10^{a}$ & $0,29 \pm 0,01^{b}$ & $0,19 \pm 0,03^{b}$ & $0,26 \pm 0,04^{b}$ & $0,28 \pm 0,01^{b}$ & 17,8 \\
\hline
\end{tabular}

Letras distintas na mesma linha indicam diferença significativa $(p<0,05)$ pelo teste de Duncan. Valores em medias \pm erro padrão C.V. (Coeficiente de variação). ND= não detectado.

*Efeito quadrático

$C 18: 2 \omega 6-Y=10,7862+0,261216 x-0,001185 x^{2}-R^{2}=70 \%$

$C 18: 3 \omega 6-Y=0,1432+0,014040 x-0,000062951 x^{2}-R^{2}=71 \%$

$\mathrm{C} 18: 3 \omega 3-Y=0,365547+0,025541 x-0,000115 x^{2}-R^{2}=71 \%$

$C 20: 4 \omega 6-Y=-0,052371+0,004357 x-0,000015514 x^{2}-R^{2}=68 \%$ 
graxos poliinsaturados. Os ácidos graxos $\gamma$ linolênico e $\alpha$-linoléico são importantes na alimentação humana, principalmente pelo fato de eles prevenirem o aparecimento de doenças cardíacas (Ribeiro, 2003).

Para oácido graxo araquidônico(C20:4 $1 \omega 6)$, a suplementação de vitamina $\mathrm{E}$ na dieta influenciou de forma quadrática $(\mathrm{p}<0,05)$, aumentando até a suplementação estimada de $140 \mathrm{mg}$ de vitamina $\mathrm{E}$ (tabela IV). O aumento da proporção de ácido graxo araquidônico

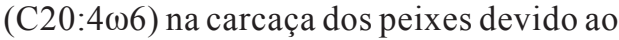
aumento de suplementação de vitamina $\mathrm{E}$, também foi relatado por Bai e Lee (1998) que observaram aumento do ácido graxo

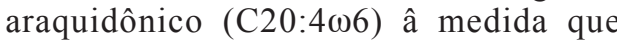
aumentou a vitamina $E$ na dieta até o nível de

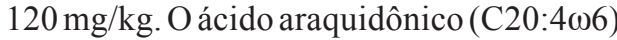
é obtido em três fontes: fosfolipídios de reserva do organismo, dieta e a partir do processo de alongamento e dessaturação do ácido linoléico (Suárez-Mahecha et al., 2002). Tocher et al. (2002) analisaram ácidos graxos no fígado e não observaram aumento do ácido araquidônico para espécies de peixes Scophthalmus maximus e Hippoglossus hippoglossus com o aumento da vitamina E na dieta. Os referentes autores observaram diferenças na quantidade de ácido araquidônico para espécie de peixe Sparus

\section{BIBLIOGRAFIA}

Almeida, N.M., Moura, J.M.L.N., Franco, R.C.N. e Bueno, M.M.R. 2006. Tocoferois do músculo dorsal e cavidade ocular do matrinxã (Brycon cephalus) da Bacia Amazônica em diferentes épocas sazonais. Ciênc. Rural, 36: 636-640.

AOAC. 1995. Official Methods of Analysis of AOAC International. P. Cunniff (ed.). $16^{\text {th }}$ ed. Arlington. vol. I e II. 469 pp.

Bai, S.C. and Lee, K.J. 1998. Different levels of dietary $\mathrm{DL}-\alpha$-tocophery affect the vitamin $\mathrm{E}$ status of juvenile Korean rockfish, Sebastes schlegeli. Aquacuaculture,113: 415-444.

Baker, R.T.M. and Davies, S.J. 1996. Changes in tissue a-tocopherol status and degree of lipid peroxidation with varying a-tocopheryl acetate inclusion in diets for the African catfish. aurata ao serem alimentadas com o nível de $1000 \mathrm{mg}$ de vitamina $\mathrm{E} / \mathrm{kg}$.

O nível do ácido graxo eicosapentaenóico EPA $(C 20: 5 \omega 3)$ foi maior $(\mathrm{p}<0,05)$ para o tratamento sem suplementação de vitamina $\mathrm{E}$ (tabela IV). Isso também foi observado no estudo de Bai e Lee (1998) ao mostrarem que no tratamento sem suplementação de vitamina $\mathrm{E}$ houve maior quantidade de ácido graxo eicosapentaenóico EPA (C20:5 23 ). Tocher et al. (2002) observaram aumento do EPA para peixes da espécie Hippoglossus hippoglossus, alimentados com níveis de vitamina E. Nesse contexto, Maia et al. (1998) encontrou valores de EPA e DHA altos em relação aos lipídios totais para algumas espécies naturais do Rio Amazonas, como 6,47 e 7,19\% para Cishla sp., 9,57 e 19,28\% para Pellona castelnaena e 9,15 e $4,46 \%$ para Liposarcus pardalis, respectivamente.

\section{CONCLUSÕES}

A dieta suplementada com vitamina $\mathrm{E}$ (vitamina E monofofatada) não influenciou o desempenho de tilápia do Nilo. Entretanto, para composição de carcaça é recomendável uma dose entre $110 \mathrm{mg} / \mathrm{kg}$ a $140 \mathrm{mg} / \mathrm{kg}$ de vitamina E.

Aquacult. Nutr., 2: 71-79.

Barreto, S.L.T. 1998. Niveis de proteina e vitamina E para matrizes de frangos de corte na fase de produção. Tese (doutorado). Escola de Veterinária. Universidade Federal de Minas Gerais, Belo Horizonte. 171 pp.

Bhujel, R.C. 2000. A review of strategies for the management of Nile tilapia (Oreochromis niloticus) broodfish in seed production systems, especially hapa-based systems. Aquaculture, 181: 37-59.

Cavalheiro, J.M.O. e Pereira, J.A. 1998. Efeito de níveis de proteína e energia em dietas no crescimento do robalo, Centropomus parallelus (Poey, 1860) em água doce. In: Congresso Aqüicultura Brasil' 98. Anais... Recife, 2: 35-40. 


\section{DESEMPENHO DE TILÁPIA DO NILO COM VITAMINAE}

Fogaça, F.H.S. and Sant'Ana, L.S. 2007. Tocopherol in the lipid stability of tilapia (Oreochromis niloticus) hamburgers. Food Chem., 105: 12141218.

Guerra, M.M.P., Evans, G. e Maxwell, W.M.C. 2004. Papel de oxidantes e anti-oxidantes na andrologia: Revisão de literatura. Rev. Bras. Reprod. Anim., 28: 187-195.

Huang, S.L., Weng, Y.M. and Huang, C.H. 2004. Lipid peroxidation in sarcoplasmic reticulum and muscle of tilapia is inhibited by dietary vitamin and supplementation. J. Food Biochem., 28: 101-111.

Kocabas, A.M. and Gatlin, D.M. 1999. Dietary vitamin $E$ requirement of hybrid striped bass (Morone chrysops female $\times M$. saxatilis male). Aquacult. Nutr., 5: 3-7.

Lovshin, L.L. 1997. Tilapia farming: a growing worldwide aquaculture industry. In: I Simpósio Sobre Manejo e Nutrição de Peixes. 1997. Anais... Piracicaba. pp. 90-98.

Maia, E.L., Rodriguez-Amaya, D. and Fraco, M.R.B. 1998. Fatty acids of the total, neutral, and phospholipids of the Brazilian freshwater fish Prochilodus scrofa. J. Food Comp. Anal., 7: 240-251.

Martins, M.L., Miyazaki, D.M. e Yamaguchi, M.F. 2008. Ração suplementada com vitaminas $\mathrm{C} e$ $E$ influencia a resposta inflamatória aguda em tilápia do Nilo. Ciênc. Rural, 38: 213-218.

Murray, T. and Andrews, J.W. 1994. Interactions of dietary $\alpha$-tocopherol, oxidized menhaden oil and ethoxyguin on channel catfish (Ictalurus punctatus). J. Nutr., 104: 1416-1431.

NRC. 1993. Nutrient requirements of fish. National Research Council. National Academy of Science. Washington, D.C. 105 pp.

Navarro, R.D., Ribeiro Filho, O.P., Ferreira, W.M.e Pereira, F.K.S. 2007. A importância da vitamina E, C e A na reprodução de peixes (Revisão de literatura). Rev. Bras. Reprod. Anim., 33: 20-25.

Paul, B.N., Sarkar, S.N. and Mohanty, S. 2004. Dietary vitamin E requirement of mirigal, Cirrhinus mrigala fry. Aquaculture, 242: 529-536.

Pearce, J., Harris, J.E. and Davies, S.J. 2003. The effect of vitamin $E$ on the serum complement activity of the rainbow trout, Oncorhynchus mykiss (Walbaum) Aquac. Nutrition, 9: 337340.

Pezzato, L.E. 1999. Alimentação de peixes-relação custo e benefício. In: XXXVI Reunião Anual Sociedade Brasileira de Zootecnia, 1999. Anais... Porto Alegre. p. 109-118.

Ribeiro, P.A.P. 2003. Perfil de ácidos graxos poliinsaturados em filés de tilápias do Nilo (Oreochromis niloticus) mantidas em diferentes condições de cultivo. (Dissertação de mestrado). Universidade Federal de Lavras. Lavras. $55 \mathrm{pp}$.

Rostagno, H.S., Albino, L.F.T., Donzele, J.L., Gomes, P.C., Ferreira, A.S., Oliveira, R.F. e Lopes, D.C. 2000. Tabelas brasileiras para aves e suínos. Composição de alimentos e exigências nutricionais. Universidade Federal de Viçosa-MG. MG, Brasil. 114 pp.

Ruff, N., Fitzgerald, R.D. Cross, T.F., Hamre, K. and Kerry, J.P. 2003.The effect of dietary vitamin E and $\mathrm{Clevel}$ on market-size turbot (Scophthalmus maximus) fillet quality. Aquacult. Nutr., 9: 91103.

Sampaio, F.G., Kleemann, G.K., Sá, M.V.C., Pereira, A.S., Barros, M.M. e Pezzato, L.E. 2004. Níveis de vitamina $\mathrm{E}$ e de selênio para pós-larvas de Macrobrachium mazonicum. Acta Sci., 26: 129-135.

SAS. 1997. Statistical Analysis System. SAS/ STAT user's guide. Version 6.0. $4^{a}$ ed. SAS, institute, Inc. North Caroline. 943 pp.

Shearer, K.D. 1994. Factors affecting the proximate composition of cultured fishes with emphasis on salmonids. Aquaculture, 119: 63-88.

Shiau, S.Y. and Shiau, L.F. 2001. Re-evaluation of the vitamin $E$ requirements of juvenile tilapia (Oreochromis niloticus $\times 0$. aureus). Anim. Sci., 72: 529-534.

STQ. 1998. Setor de Tecnologia Química. Metilação de óleos e gorduras. Fundação Centro Tecnológico de Minas Gerais (CETEC). Belo Horizonte. $2 \mathrm{pp}$.

Suárez-Mahecha, H., Francisco, A., Beirão, L.H., Block, J.M., Saccol, A. e Pardo-Carrasco, S. 2002. Importância de ácidos graxos poliinsaturados presentes em peixes de cultivo e de ambiente natural para a nutrição humana. Bol. Inst. Pesca, 28: 101-110.

Tachibana, L., Portz, L. e Cyrino, J.E.P. 2000 Influência do dimetilsulfoxido (DMSO) na reversão sexual de tilápias do Nilo (Oreochromis niloticus) tratadas com $17 \alpha \alpha$-metiltestosterona. In: VI Simpósio Brasileiro de Aqüicultura 2000. 


\section{NAVARRO, FERREIRA, RIBEIRO FILHO, VELOSO, FONTES E SILVA}

Anais.... Florianópolis. não paginado, CD ROM. Tocher, D., Mourente, G., Van Dereecken, A., Evjemo, J., Diaz, E., Bell, J.G., Geurden, I. Lavens, P. and Olsen, Y. 2002. Effects of dietary vitamin $E$ on antioxidant defence mechanisms of juvenile turbot Scophthalmus maximus L., halibut Hippoglossus hippoglossus L. and sea bream Sparus aurata L. Aquacult. Nutr., 8: 195-207.

Thakur, A.I. and Srivastava, U.S. 1996. Vitamin-E Metabolism and its application. Nutr. Res., 16: 1767-1809.

Watanabe, T. and Takashima, F. 1977. Effect of $\alpha-$ tocopherol deficiency on carp. VI. Deficiency symptoms and changes of fatty acid and triglyceride distribuitions in adults carp. Bull. Jap. Soc. Sci. Fish., 43: 819-830.

Wilson, R.P., Bowser, P.R. and Poe, W.E. 1984. Dietary vitamin $E$ requirement of fingerling channel catfish. J. Nutr., 114: 2053-2058.

Wing-Keong, Ng, Wang, Y., Ketchimenin, P. and Yuen, K. 2004. Replacement of dietary fish oil with palm fatty acid distillate elevates tocopherol and tocotrienol concentrations and increases oxidative stability in the muscle of African catfish, Clarias gariepinus. Aquaculture, 233: 423-437.

Archivos de zootecnia vol. 59, núm. 226, p. 194. 